\title{
Infection Prevention and Control Practices in Public Health Facilities Compared to the Confessional and Private Ones
}

\author{
Ndipowa James Attangeur Chimfutumba ${ }^{1, ~}$, Yongabi Kenneth Anchang², Dismas Ongore ${ }^{3}$, \\ Nyabola Lambert ${ }^{3}$ \\ ${ }^{1}$ Department of Nursing, Institute of Medicine and Biomedical Sciences, Cameroon Christian University, Bali, Cameroon \\ ${ }^{2}$ Phytobiotechnology Research Foundation Institute, Catholic University of Cameroon, Bamenda, Cameroon \\ ${ }^{3}$ School of Public Health, Department of Community Health, University of Nairobi, Kenya
}

\section{Email address:}

ndipowajames@gmail.com (N. J. A. Chimfutumba), yongabika@yahoo.com (Y. K. Anchang), dongore2000@yahoo.co.uk (D. Ongore), lnyabola@uonbi.ac.ke (N. Lambert)

\section{To cite this article:}

Ndipowa James Attangeur Chimfutumba, Yongabi Kenneth Anchang, Dismas Ongore, Nyabola Lambert. Infection Prevention and Control Practices in Public Health Facilities Compared to the Confessional and Private Ones. Science Journal of Public Health.

Vol. 3, No. 6, 2015, pp. 865-872. doi: 10.11648/j.sjph.20150306.21

\begin{abstract}
Infection prevention, control and health promotion have been a serious challenge to the public health sector in Cameroon in general and in the Bamenda health district in particular. This has led to an upsurge of many infectious diseases and epidemics in recent times. It has been aggravated by the advent of the Ebola hemorrhagic disease in neighboring countries and other existing epidemics such as Poliomyelitis, Cholera, Tuberculosis, HIV/AIDS, and Measles. This survey compares the infection prevention, control and health promotion practices in public health facilities with those in the confessional and private health facilities. From the analyses, the number of males with sound practices doubled that of females, thus proving to be of statistical significance. Denominational or confessional health facilities equally recorded a high percentage of sound practice followed by private health facilities and then government-owned health facilities. There is also a significant relationship between "level of knowledge" and "infection prevention, control and health promotion practice" from this study.
\end{abstract}

Keywords: Infection, Prevention, Practices, Control, Health, Facilities

\section{Introduction}

In the Bamenda health district there has been an upsurge of many infectious diseases and epidemics in recent times, aggravated by diseases such as Poliomyelitis, Cholera,
Tuberculosis, HIV/AIDS, Measles etc. This notwithstanding, it is worth of note that infection transmission can be quite common, understandably due to patient/client care activities in the hospital milieu.

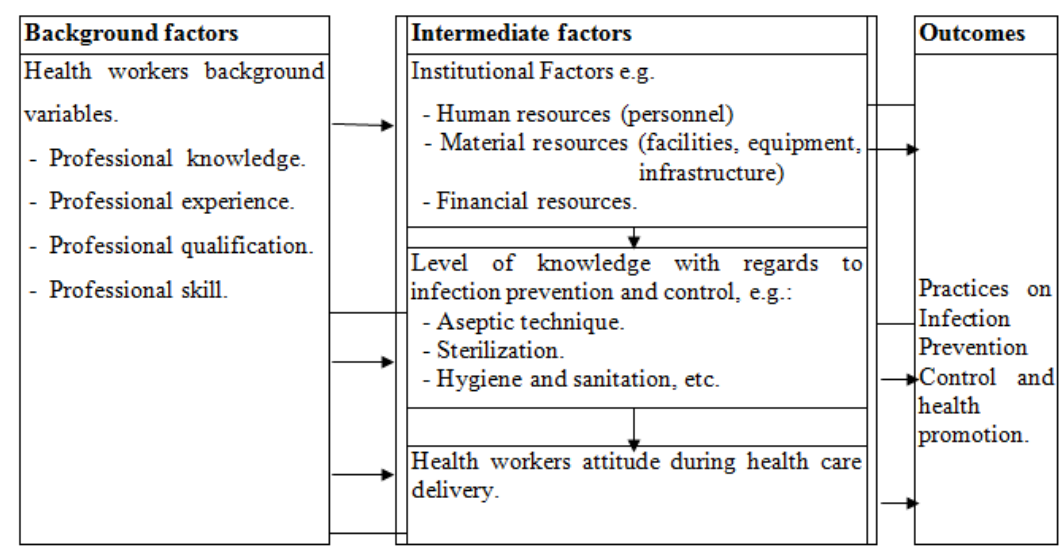

Fig. 1. Conceptual Framework. 


\section{Conceptual Framework}

There exists interrelationship between the background factors, intermediate factors and the outcomes. The professional knowledge, professional experience, professional qualification and professional aptitude greatly rely on the institutional resources in order for the outcomes to be yielded in terms of infection prevention, control and health promotion. Meanwhile the institutional factors such as human, material and financial resources will facilitate the acquisition of professional knowledge and aptitudes by the personnel especially as concerns aseptic technique, sterilization, hygiene and sanitation. This acquisition of knowledge and aptitudes is also a positive reinforcement and motivating factor which greatly influences the attitude of the personnel in terms of their infection prevention, control and health promotion practices (Fig. 1)

\section{Methodology}

\subsection{Study Design}

It was a descriptive, cross sectional, comparative and analytic survey of the factors influencing infection prevention, control and health promotion practices in public, private and denominational health facilities in the Bamenda Health District. There was a cross sectional survey of the health units, health workers, key informants interview, an observation guide, a focus group discussion and also openended questions in the last part of the health workers interview to help explain or validate the findings.

The following variables were considered:

1). Independent Variables:

- The available infrastructure. This was observed using an observation checklist.

- Amenities and facilities for infection control. This was surveyed through observation and also personnel interview.

- Health workers knowledge on infection prevention and control. This was assessed by use of structured and semi-structured questionnaire and also direct observation with the aim of determining the level of knowledge of the personnel and their performance with regard to selected infection prevention and control procedures.

- The problems (constraints) encountered by the health workers during infection prevention and control. This was assessed through a structured questionnaire, Focus Group Discussion and interview.

2.) Dependent Variables:

- Infection prevention practices.

- Infection control practices.

\subsection{Sampling}

Selection of health facilities:
Multistage sampling with stratification was done to select the health facilities under study. This method was preferable because the health facilities in the Bamenda health district are made of three strata, namely: the Public Health Facilities, 12 in number; the Mission Health Facilities, 6 in number; and the Private Health Facilities, 7 in number. A total of 15 out of the 25 available, functional and approved health facilities in the Bamenda Health District were selected randomly and proportionately as follows:

- Public Health Facilities $=7$.

- Mission Health Facilities = 4, and.

- Private Health Facilities $=4$.

This was to ensure an adequately representative sample for the study.

\subsection{Selection of Key Informants}

Purposive sampling was done to select 25 key informants from 15 selected health facilities.

\subsection{Selection of Focus Group Discussion (FGD) Members}

The participants for the FGD were basically made of ward charges, coordinators and supervisors of services / units and who were not served the key informants questionnaire. Systematic random sampling was used to select 10 participants out of the prepared list of 15 ward charges and coordinators / supervisors of services and units in the selected health facilities. These constituted the membership for the Focus Group Discussion.

\subsection{Sample Size Determination} [9]:

The sample size was determined by the Fisher's formula

$$
n=\frac{Z^{2} P(1-P)}{d^{2}}
$$

Where:

$n=$ The desired sample size where the study population is equal to or greater than 10,000 .

$Z=$ Standard normal deviate corresponding to $95 \%$ level of confidence $(=1.96)$.

$p=$ Estimated prevalence of characteristic of interest (unsound practices) $(=0.5)$.

(Since that of the Bamenda Health District is not known). $d=$ Level of precision (set at $\pm 5 \%$ ).

Therefore,

$$
n=\frac{1.96 \times 1.96 \times 0.5 \times 0.5}{0.05 \times 0.05}=384.16=384 \text { persons }
$$

Meanwhile, in this study, the study population was 531 health workers, distributed as follows:

(a) Government health units $=248$ H.Ws $(46.7 \%)$

(b) Denominational health units $=190$ H.Ws $(35.8 \%)$

(c) Private health units $=93$ H.Ws $(17.5 \%)$

To obtain the sample size for this study the adjustment formula was used as follows: 


$$
\mathrm{T} n f=\frac{n}{1+\frac{n-1}{N}}=\frac{384}{1.72}=221.51
$$

Therefore the sample size, $n f=222$ persons.

The samples were therefore allocated proportionately according to the above proportions as follows:

(a) Government Health Units $(46.7 \%)=103.7=104$ persons.

(b) Denominational Health Units $(35.8 \%)=79.5=79$ persons.

(c) Private Health Units $(17.5 \%)=38.9=39$ persons.

N.B. This sample size was slightly increased due to the situation found on the field. Where there was an operating theatre and / or a blood bank, 2 more participants were selected from each of the units during the working shifts by convenience sampling. That is why the sample size finally came up to 232 persons instead of the 222 previewed.

\section{Relationships Between Variables}

The main objective of this analysis was to compare the performance of tasks and procedures in infection prevention and control in the confessional health facilities with the other health facilities with regards to the conventionally acceptable standards.

The following variables were considered:

(a) Independent Variables

Sex, Type of Health Facility, Designation of Staff, Locality, Type of Health Sector, Level of Knowledge and attitude.

(b) Dependent variable

Sound Practice of infection prevention, control and health promotion.

Both bivariate and multivariate analyses were performed.

(c) Results of bivariate analyses

The results of bivariate analyses of Independent Variables by sound practice are presented in the following table.

Table 1. Results of Bivariate Analysis for Relationships between Independent Variables and Sound Practice.

\begin{tabular}{|c|c|c|c|c|}
\hline Characteristic & Number with sound practices & $\%$ & Chi. Square & P-Value. \\
\hline \multicolumn{5}{|l|}{ Sex } \\
\hline Male $(n=45)$ & 18 & 40.0 & \multirow{2}{*}{4.808} & \multirow{2}{*}{ * 0.028} \\
\hline Females $(\mathrm{n}=55)$ & 11 & 20.0 & & \\
\hline \multicolumn{5}{|l|}{ Type of health facility } \\
\hline District Hospital (n=18) & 7 & 38.9 & \multirow{4}{*}{6.223} & \multirow{4}{*}{0.101} \\
\hline Provincial hospital $(\mathrm{n}=22)$ & 2 & 9.1 & & \\
\hline Health centre $(n=34)$ & 10 & 29.4 & & \\
\hline linic $(n=26)$ & 10 & 38.5 & & \\
\hline \multicolumn{5}{|l|}{ Designation of staff } \\
\hline Doctor $(n=9)$ & 8 & 88.9 & & \\
\hline Nurse $(\mathrm{n}=29)$ & 12 & 41.4 & \multirow{4}{*}{27.147} & \multirow{4}{*}{$* 0.000$} \\
\hline Nurse / Midwife $(\mathrm{n}=22)$ & 4 & 18.2 & & \\
\hline Laboratory Technician $(\mathrm{n}=23)$ & 4 & 17.4 & & \\
\hline Theatre Attendant / Nurse Aid $(\mathrm{n}=17)$ & 1 & 5.88 & & \\
\hline \multicolumn{5}{|l|}{ Locality } \\
\hline Rural (n=19) & 9 & 47.4 & \multirow[b]{2}{*}{3.844} & \multirow[b]{2}{*}{ *0.05 } \\
\hline Urban: $(\mathrm{n}=81)$ & 20 & 24.7 & & \\
\hline \multicolumn{5}{|l|}{ Type of Health Sector } \\
\hline Government $(\mathrm{n}=62)$ & 12 & 19.4 & \multirow{3}{*}{13.329} & \multirow{3}{*}{$* 0.001$} \\
\hline Mission $(n=17)$ & 11 & 64.7 & & \\
\hline Private $(n=21)$ & 6 & 28.6 & & \\
\hline \multicolumn{5}{|l|}{ Level of knowledge } \\
\hline Adequate $(\mathrm{n}=59)$ & 22 & 37.3 & \multirow{2}{*}{4.801} & \multirow{2}{*}{$* 0.028$} \\
\hline Inadequate $(\mathrm{n}=41)$ & 7 & 17.1 & & \\
\hline \multicolumn{5}{|l|}{ Attitude } \\
\hline \multicolumn{5}{|c|}{ 1. Feeling about Infection Prevention and Control Standards. } \\
\hline Positive feeling about standards $(\mathrm{n}=28)$. & 13 & 46.4 & \multirow{3}{*}{5.737} & \multirow{3}{*}{ * 0.026} \\
\hline Negative feeling about standards $(\mathrm{n}=72)$. & 16 & 22.2 & & \\
\hline \multicolumn{3}{|l|}{ 2.Level of Motivation. } & & \\
\hline Decreased $(n=37)$ & 7 & 18.9 & \multirow{3}{*}{2.967} & \multirow{3}{*}{0.227} \\
\hline Not changed $(\mathrm{n}=58)$. & 20 & 34.5 & & \\
\hline Increased $(n=5)$ & 2 & 40.0 & & \\
\hline
\end{tabular}

* Statistically significant.

From the analyses, forty percent of the males had sound practice as against $20 \%$ for the females. The difference was found to be statistically significant $(p=0.028)$ implying that there is a statistically significant relationship between gender and practice among the health workers.

It was demonstrated that a large percentage $(88.9 \%)$ of doctors had sound practice followed to a lesser extent by nurses, midwives, laboratory technicians, nursing aids/theatre 
attendants. This implies a highly significant association between designation of staff and practice $(\mathrm{p}=0.000)$.

Sound practice was recorded more in the health facilities located in the rural areas $(47.4 \%)$ as compared to $24.7 \%$ in those located in the urban areas. However, there was no statistically significant relationship between locality and infection prevention and control practice $(\mathrm{p}=0.05)$.

The largest proportion with sound practice was recorded in the denominational or confessional health sector $(64.7 \%)$, followed by the private sector $(28.6 \%)$ and then the government sector (19.4\%). The differences in performance among the three types of health sector were found to be statistically significant. Therefore, there was statistically significant relationship between the health sector and infection prevention and control practice $(p=0.001)$.

In relation to other aspects of the study, it was recorded that there was a significant relationship between attitude and infection prevention and control practice $(\mathrm{p}=0.026)$; there was a significant relationship between level of knowledge and infection prevention and control practice $(p=0.028)$.

When the independent variables were regressed against level of performance (satisfactory or unsatisfactory), it was noticed that the other variables, namely, Sex, Type of Health Facility, Locality, Level of Knowledge and Attitude were not significantly associated with infection prevention control and health promotion practices in the Bamenda Health District.

(d) Results of Multivariate Analysis (i.e. Logistic Regression)

The independent variables were regressed against level of performance (satisfactory or unsatisfactory) and the results are presented in Table 2.

Following Logistic regression analysis, type of health sector and designation of staff were the two variables found to have statistically significant effect on sound practices regarding infection prevention, control and health promotion.

Table 2. Analysis of the Relationship between Independent Variables and Level of Practice.

\begin{tabular}{|c|c|c|c|c|c|c|}
\hline & B & S.E. & Wald's Statistic & Df & Sig. & Odds Ratio. \\
\hline Type of sector & & & 10.690 & 2 & $* 0.005$ & \\
\hline Government & -0.509 & 0.806 & 0.323 & 1 & 0.570 & 0.601 \\
\hline Mission & 2.617 & 0.986 & 7.049 & 1 & 0.008 & 13.692 \\
\hline Designation & & & 14.947 & 4 & $* 0.005$ & \\
\hline Doctor & 5.844 & 1.811 & 10.410 & 1 & $* 0.001$ & 345.061 \\
\hline Nurse & 2.495 & 1.274 & 3.838 & 1 & 0.050 & 12.122 \\
\hline Nurse / Midwife & 1.713 & 1.366 & 1.574 & 1 & 0.210 & 5.548 \\
\hline Yrs. since basic training & 0.013 & 0.020 & 0.431 & 1 & 0.512 & 1.013 \\
\hline Level of Knowledge & -0.288 & 0.705 & 0.167 & 1 & 0.683 & 0.750 \\
\hline Attitude & & & 1.327 & 2 & 0.515 & \\
\hline Feeling about Inf. Prev. & -0.462 & 1.345 & 0.118 & 1 & 0.731 & 0.630 \\
\hline Level of motivation & -1.352 & 1.480 & 0.835 & 1 & 0.361 & 0.259 \\
\hline Constant & -2.454 & 2.027 & 1.466 & 1 & 0.226 & 0.086 \\
\hline
\end{tabular}

\section{Discussion}

This discussion lays emphasis on key findings from those areas and facets that can be improved upon or changed, given the limited resources, with maximum impact. It is presented according to the specific objectives of the study as follows:

(1) To assess the knowledge of the health workers with respect to infection prevention, control and health promotion practices.

(2) To compare the performance of tasks and procedures in infection prevention control and health promotion in the confessional health facilities with the others with regard to the conventionally acceptable standards.

\section{Key Findings}

\subsection{Knowledge of Health Workers}

A survey by Bosse (2001) in Southern Tanzania showed that among other factors, mission referral hospitals performed better than the government hospitals and it was because there was a high possibility for internal refresher training not only for reasons of poor performance but also for better performance, as well as to boost staff morale [5].

This internal refresher training coupled with a possibility to upgrade nursing aides to nurses or midwives served like a positive reinforcement and could improve the performance levels of the staff.

The staff in the Bamenda health district also needs this type of positive reinforcement and motivation.

Majority of the respondents knew that HIV/AIDS, Ebola Fever, hepatitis B, syphilis, malaria and tetanus could be transmitted by contact with infected blood, unsafe injection or unsterile procedure.

In another study among Angolan soldiers the results showed more consistent improvement in knowledge, motivation and behavior in terms of HIV, malaria and other infection transmission in 2006 after refresher courses and knowledge update $[18 ; 22]$.

The situation has not changed in Cameroon since for more than 4 years no refresher training or knowledge update has been carried out in the Bamenda health district. After the analysis of the level of knowledge of the participants under study, $37.3 \%$ of the 59 health workers with adequate knowledge had sound practice while $17.1 \%$ of the 41 health workers with inadequate knowledge had sound practice. This means that a lot still has to be done to harmonize knowledge 
with performance, especially in a situation where there is no Infection Prevention and Control Unit found in any of the health units under study.

From the responses all the respondents were taught hand washing and surgical scrub during their basic training as an infection prevention measure though most of them could neither describe it nor carry it out satisfactorily.

It is worth of note that hand washing and surgical scrub are the very first and basic tasks to be done to ensure asepsis and infection prevention and control before and after attending to every patient especially when carrying out medico-surgical procedures. Unfortunately, it was commonplace to find health personnel of various grades carry out medico-surgical procedures on patients and even do complete ward rounds without thinking of washing their hands, let alone to think of hand washing before and after attending to each patient / client. Slightly over a third of health personnel were unable to describe the technique of hand washing and surgical scrub and several other procedures satisfactorily. It should therefore be encouraged and reinforced for all staff since excellence in the psychomotor greatly depends on the knowledge and attitude acquired.

Reminders of the importance of hand washing as a simple and effective means of preventing the spread of infection abound. For example, in Boston (USA), National Public Radio announcements around Christmas Eve include reminders for priests to wash their hands thoroughly before they handle communion bread, in order to prevent the spread of seasonal infections, particularly influenza. Similarly, in the Far East, public health advice concerning the latest outbreaks of viral infections such as SARS pointed the lay person in the direction of the simple practice of hand washing with soap and water, especially in children's day care centers, preschools, schools, shopping malls and other public places. Hospital nurses, doctors and consultants are rarely mentioned in public as likely vehicles of the spread of infection in health care settings; and yet they are, if they fail to wash their hands routinely and thoroughly.

In the USA, patients are advised to demand that their doctors wash their hands before touching them [1].

In a research carried out in Karen Hospital, with main objective of assessing quality care with respect to hand washing, the results showed that only $30 \%$ of health workers routinely washed their hands between patient contact. The study revealed genuine interest in training and the need to reward good practice in order to motivate health workers. Educational intervention and technical training resulted in significant improvements in health workers' compliance with hospital infection prevention standards. Patient satisfaction with health workers' hygiene practices also improved significantly [22]. In conclusion hospital-based health workers' hand washing practice needs to improve globally. The study confirms that both cognitive and technical inputs are necessary for infection prevention, it is not an 'either-or' proposition. Promotion of hand washing is also relevant to clinics and health stations which do not accommodate in-patients.

Proper hand hygiene is the single most important infection prevention and control practice. As a matter of fact the health workers in the Bamenda health district still leave more to be desired in terms of hand washing. This ought to be a rule of thumb.

With regards to attitude towards practice, only $46.4 \%$ of the 28 health workers who felt that infection prevention and control practices were within acceptable standards had sound practice. On the other hand, $22.2 \%$ of the 72 health workers who felt that infection prevention and control practices were not within acceptable standards had sound performance.

Angola, in Southern Africa, is illustrative of many resource-limited countries in Sub-Saharan Africa. Several years after the end of a 27-year civil war, the country is rebuilding under the weight of extreme poverty and a destroyed infrastructure. The leadership of the Angolan military has expressed concern that the rate of HIV infection may quickly increase as post-war mobility and cross-border traffic increase. Thus, there is both the need and the political will for an effective HIV prevention intervention in the Angolan military [13].

In sum, this study presents evidence that militaries, even in resource-limited post-conflict countries, can provide effective infection prevention and control to their soldiers.

It is hoped that if the leadership of the Bamenda health district in particular and that of the North West Region in general had this type of concern then infection prevention, control and health promotion especially concerning HIV/AIDS and Ebola Hemorrhagic Fever will register significant successes.

Important to remember that the respondents proposed some solutions aimed at improving infection prevention, control and health promotion in the Bamenda health district and the prominent ones were:

Supply of adequate equipment and materials; Recruitment and employment of more qualified staff; Update of knowledge of personnel through in-service training; Refresher courses and seminars; Staff motivation through salary and incentives; and Improvement of pipe borne water.

If these solutions, including the less prominent ones, were addressed in order of priority then there would ultimately be significant improvement in the quality of health care, especially infection prevention, control and health promotion.

One of the reasons for health workers in Cameroon maintaining their poorly paid government jobs was the absence of a strict supervision and monitoring system [12]. Without proper supervision and monitoring, personnel performance will be difficult to know; hence fruitful correction can hardly be made. It is believed that even the most well trained workers in any sector if not well supervised and monitored cannot perform to the maximum expected standard.

In the Bamenda Health District this was lacking. Nevertheless, despite all the other shortcomings, with the available resources it could be done with significant impact on the infection prevention, control and health promotion practices in the immediate / short term. For example: health workers not washing their hands after attending to each 
patient; not being able to do surgical scrub; not respecting aseptic technique in injection giving; laboratory technicians wearing the same pair of gloves and attending to several patients, just to name a few. These issues could be sorted out at short term.

With the advent of Ebola Hemorrhagic Disease, infection prevention, control and health promotion should be given pride of place and sound practice of this important aspect of health care is mandatory both in all health facilities and also in the community. The following measures are therefore very crucial:

\subsection{Hand Washing}

Hand washing is the single most-important infection prevention and control practice as already mentioned above. It is vital that health care staff follow hand washing protocols that are appropriate for their clients and facility.

\subsection{Protective Barriers}

Examples of protective barriers include gloves, masks, eyewear, gowns, boots and plastic aprons. The appropriate barrier should be used when blood, secretions or body fluids are likely to come in contact with the health worker's skin or mucous membranes, or could penetrate clothing.

\subsection{Care of Equipment}

This involves the appropriate disposal of waste, contaminated laundry and sharps; and the cleaning, sterilization and disinfection of equipment, instruments and devices. Health staff should follow manufacturer and facility protocols in all instances.

\subsection{Health Practices of the Health Care Providers}

Health care providers who believe they have been contaminated with an infectious agent should contact their primary health care provider or an occupational health department for follow-up and advice. They should assess the risk of transmitting the infectious agent to others and take appropriate precautions.

In the United States, HIV prevention programmes have focused on people at risk for HIV, as well as those who are already infected. Given that additional HIV prevention efforts in the USA are needed to reduce the incidence of the disease, the Center for Disease Control (CDC) launched a new strategy for HIV prevention in 2001 called Sero-status Approach to Fight the Epidemic (SAFE) [2].

This was designed to increase:

1. The number of persons who know their status;

2. The use of health care and preventive services;

3 . High quality care and treatment services; and.

4. The number of individuals with HIV who adopt and sustain HIV risk reduction behavior.

Considering the various flaws and inadequacies observed during the research project, it would be very right to say that the infection prevention, control and health promotion practices in the medico-surgical units of the Bamenda health district are unsatisfactory compared to the required conventionally accepted standards, even though the confessional health facilities are putting on some appreciable efforts to that effect.

Generally the performance of the health workers in terms of infection prevention and control was averagely good but a lot still has to be done to meet up with the recommended conventional standards.

\section{Conclusions}

The acquisition of knowledge coupled with change of attitude and practice by health service providers with regards to infection prevention, control and health promotion will significantly reduce the prevalence of HIV/AIDS, Measles, Cholera and other infections in the Bamenda health district.

Our Null Hypothesis $\left(\mathrm{H}_{0}\right)$ is accepted - Poor and inadequate infection prevention, control and health promotion practices exist in health facilities in the Bamenda Health District, contributing very significantly to the high prevalence rates of HIV/AIDS, Measles, Cholera and other infections. This is marked by:

- Unsatisfactory or ineffective sterilization of equipment and materials; poor garbage and hospital refuse disposal, unsatisfactory aseptic technique used in most of the health facilities and unsatisfactory blood transfusion services.

- Following Logistic regression analysis, type of health sector and designation of staff were the two variables that had statistically significant effect on the level of practice regarding infection prevention, control and health promotion.

- The confessional health facilities were significantly distinguished for sound practice.

- It would be right to also conclude that the infection prevention, control and health promotion practices in the Bamenda Health District do not meet up with the required conventionally acceptable standards. Serious improvement is therefore mandatory.

\section{Proposals and Recommendations}

(a) Immediate / short term

1) Hand washing / surgical scrub should be reinstated and reinforced by health authorities especially when carrying out medico-surgical procedures and ward rounds.

2) The authorities concerned should supply adequate materials and functional equipment such as Autoclaves; Boilers; Hot Air Oven and other modern sterilization equipment, repair those that are broken down and ensure constant maintenance.

3) More modern methods of sterilization such as chemical sterilization, steaming, internal fumigation and infrared sterilization should be taught and made available to the staff of various health facilities.

4) The relevant authorities should re-enforce supervision. 
There has to be some commitment to surveillance and monitoring to check and prevent infection transmission.

(b) Moderate term

1) A health district census or an inventory of incinerators and refuse disposal pits should be made and provision made in health facilities where there are no incinerators and refuse disposal pits.

2) Authorities of the Health District and Regional Delegation should set up an operational and sustainable system of infection control. This entails setting up an infection control unit in each heath facility. None exists for now.

3) The relevant authorities should create and sustain a District Blood Transfusion Coordination and Control service for effective and scrupulous supervision and control of the use of blood products.

4) The government authorities should sign a Memorandum of Undertaking (MOU) with the confessional health facilities and the private ones allowing for exchange working visits in order to harmonize and standardize performance of health care delivery.

(c) Long term

1) Refresher courses, seminars, workshops and in-service training should be made available to the personnel regularly in order to update their knowledge on infection prevention, control and health promotion.

2) There is absolute need for the government to decentralize the health care delivery system. This will enable the public health authorities take more concerted decisions and actions in health care delivery matters at various levels.

\section{Further Research Work}

(1) A similar research project is recommended to be carried out covering the whole North West Region and possibly more regions of the country so that the results could be more inferential.

(2) A study is also recommended on blood transfusion safety with emphasis on HIV infection prevention and control in the Bamenda Health District.

\section{References}

[1] American College of Preventive Medicine. U.S. Preventive Services Task Force Recommendations Human Immunodeficiency Recommendations. Available at: //www.acpm.org/clinical.htm.

[2] Berenson, A.S. (2005) Control of Communicable Diseases Manual. American Public Health Association, Washington, D.C. 16th Edition.

[3] Bing et al (2005) HIV Preventive and Control Interventions by Angolan soldiers. Report available at http://www.pubmedcentral/pubmedcentral.htm Accessed April 10, 2010.

[4] Boonstra et al (2002) Adherence to health care guidelines. Comparative survey of the performance of formally well trained staff and non - trained or informally trained staff in Botswana. Article published at http://www.pubmedcentral/pubmedcentral.htm Accessed On April 13, 2010. 5. Bosse (2001) Factors Influencing the Performance of Health Staff in Southern Tanzania. Article published at http://www.pubmed-central/pubmedcentral.htm Accessed April $10,2010$.

[5] Butler et al (2004) Transmission of Human Immunodeficiency Virus. Contaminated blood transfusion. Model - based estimates, 78: 801-807.

[6] CIA (2002) Carte Institutionelle et Administrative du Cameroun. Système exécutif, judiciaire et législatif. Edition clé.

[7] Eddleston, M., Robert Davidson et al (2005) Oxford Handbook of Tropical Medicine. Oxford University Press, Second Edition, 42-115.

[8] Fisher et al (1998) Interpretation and Application of Biostatistics. Medicine; Statistics. Oxford scientific publications. $5^{\text {th }}$ Edition. 123-127; 414-417.

[9] Gayraud, M., Lotholary, 0.(2006) Maladies Infectieuses VIH/SIDA 11 Masson, 4eme Edit, Pp 179-234.

[10] Gumodoka et al (2001) Injection Practices in Mwanza Region, Tanzania: prescription, patient demand and sterility. Tropical medicine and international health. 874-880.

[11] Israr et al (2000) Health Care Delivery Systems. The case of Cameroon. SOPECAM, Cameroon tribune, Jn.17, 2000. 13 15.

[12] Joint United Nations Programme on HIV/AIDS. Available at www.unaids.org Accessed Dec. 2006.

[13] Kipata, B., Ngaly, B. et al (2008) Human Immunodeficiency Virus Infection Among Employees in an African Hospital. N. Engi J Mod 1988 319: 1123-1127.

[14] Kuhu L. and Stein Z.A. (2005): Mother-to-Child HIV Transmission Timing and Determinants. Obst. Gynaecol. Clin. North Am. 9th Edition. 1-29.

[15] Kwiek J.J; Mwapasa, V. et al. (2006): Maternal-fetal Micro transfusion and HIV-1 Mother-to-Child Transmission in Malawi. Vol.3, Issue 1/10.

[16] Ministry Of Health, Kenya (2007) National Standards and Guidelines on Injection Safety And Medical Waste Management. NASCOP - Nairobi, Kenya. First Edition.

[17] PubMed (2009) Infection Prevention and Control. Publication No.41002. http://www.pubmed- central/pubmedcentral.htm.

[18] Republic of Kenya, MOH. (March 2008) HIV Prevention Messages for People Living WithHIV/AIDS: A tool for health care providers in clinical settings. NASCOP. Trainers Manual.

[19] République du Cameroun. Ministère de la santé publique. Cadre conceptuel du District de la santé viable au Cameroun, pp 6.

[20] République du Cameroun (1998 - 2008) Plan National de Développement Sanitaire. Plan Stratégique. Edition Clé. SOPECAM, Yaoundé. Pp 115-136.

[21] Rigbe S. \& Almedom A.M. (2005) Assessing Quality Health Care with respect to Hand washing. Pub Med Publication. Available at http://www.pubmed-central/pubmedcentral.htm. 
[22] Sunder Lal; Adarsh; Pankaj (2007) Textbook of Community Medicine - Preventive and Social Medicine, CBS Publishers \& Distributor New Delhi, Bangalore. 322-327, 336 -349.

[23] Joint United Nations Programme on HIV/AIDS. Available at www.unaids.org Accessed Dec. 2006.
[24] WHO (2006) Guide to Planning Health Promotion for AIDS Control. Global Programme On AIDS, Geneva. 